\title{
Systematic review of interventions to improve patient uptake and completion of pulmonary rehabilitation in COPD
}

\author{
Arwel W. Jones ${ }^{1}$, Abigail Taylor ${ }^{1}$, Holly Gowler ${ }^{1}$, Noel O’Kelly², Sudip Ghosh ${ }^{2,3}$ \\ and Christopher Bridle ${ }^{1}$
}

Affiliations: ${ }^{1}$ Lincoln Institute for Health, University of Lincoln, Lincoln, UK. ${ }^{2}$ Community Health Services, Leicestershire Partnership NHS Trust, Leicester, UK. ${ }^{3}$ Faculty of Health and Life Sciences, De Montfort University, Leicester, UK.

Correspondence: Arwel Jones, Lincoln Institute for Health, University of Lincoln, Brayford Pool, Lincoln LN6 7TS, UK. E-mail: arjonesQlincoln.ac.uk

ABSTRACT Pulmonary rehabilitation is considered a key management strategy for chronic obstructive pulmonary disease (COPD), but its effectiveness is undermined by poor patient uptake and completion. The aim of this review was to identify, select and synthesise the available evidence on interventions for improving uptake and completion of pulmonary rehabilitation in COPD.

Electronic databases and trial registers were searched for randomised trials evaluating the effect of an intervention compared with a concurrent control group on patient uptake and completion. The primary outcomes were the number of participants who attended a baseline assessment and at least one session of pulmonary rehabilitation (uptake), and the number of participants who received a discharge assessment (completion).

Only one quasi-randomised study ( $\mathrm{n}=115$ ) (of 2468 records identified) met the review inclusion criteria and was assessed as having a high risk of bias. The point estimate of effect did, however, indicate greater programme completion and attendance rates in participants allocated to pulmonary rehabilitation plus a tablet computer (enabled with support for exercise training) compared with controls (pulmonary rehabilitation only).

There is insufficient evidence to guide clinical practice on interventions for improving patient uptake and completion of pulmonary rehabilitation in COPD. Despite increasing awareness of patient barriers to pulmonary rehabilitation, our review highlights the existing under-appreciation of interventional trials in this area. This knowledge gap should be viewed as an area of research priority due to its likely impact in undermining wider implementation of pulmonary rehabilitation and restricting patient access to a treatment considered the cornerstone of COPD.

@ERSpublications

A major knowledge gap exists on interventions to improve patient uptake and completion of pulmonary rehabilitation http://ow.ly/TA1E306iJRB

Cite this article as: Jones AW, Taylor A, Gowler H, et al. Systematic review of interventions to improve patient uptake and completion of pulmonary rehabilitation in COPD. ERJ Open Res 2017; 3: 00089-2016 [https://doi.org/10.1183/23120541.00089-2016].

This article has supplementary material available from openres.ersjournals.com

Received: Aug 102016 | Accepted after revision: Nov 062016

Support statement: We acknowledge the University of Lincoln Research Investment Fund for funding the Lincolnshire Joint Research Agenda on COPD. Funding information for this article has been deposited with the Open Funder Registry.

Conflict of interest: None declared.

Copyright $\odot$ ERS 2017. This article is open access and distributed under the terms of the Creative Commons Attribution Non-Commercial Licence 4.0. 


\section{Introduction}

Chronic obstructive pulmonary disease (COPD) is characterised by persistent and progressive airflow limitation, with acute exacerbations of symptoms (dyspnoea, cough and sputum production) and comorbidities contributing to the overall severity of the disease [1]. Globally, COPD is now the third leading cause of mortality [2] and the second leading cause of disability-adjusted life-years lost [3]. In the UK, mortality from COPD is almost double the European Union average, and has a high social and economic burden as a leading cause of emergency admissions to hospital [4].

Pulmonary rehabilitation is defined as a "comprehensive intervention based on a thorough patient assessment followed by patient-tailored therapies, which include, but are not limited to, exercise training, education and behavior change, designed to improve the physical and psychological condition of people with chronic respiratory disease and to promote the long-term adherence to health-enhancing behaviors" [5]. Pulmonary rehabilitation triggers moderate-to-large and clinically significant effects on maximal and functional exercise capacity, dyspnoea and fatigue, emotional function, and sense of control that COPD patients have over their condition [6]. Our confidence in the estimate of these effects is unlikely to change with any further randomised controlled trials comparing pulmonary rehabilitation and conventional care in COPD [7]. Furthermore, pulmonary rehabilitation delivered in the acute setting (post-exacerbation of COPD) also reduces the risk of hospital readmissions and mortality [8]. Hence, national guidelines recommend all patients with stable COPD and exercise limitation, due to breathlessness, should be referred to pulmonary rehabilitation or should start a pulmonary rehabilitation programme within 4 weeks of discharge from a hospital admission due to an acute exacerbation of COPD [9].

Although the beneficial effects of pulmonary rehabilitation are well established, patient uptake and completion of the treatment remains poor. A recent clinical audit of pulmonary rehabilitation services in the UK reported that only $42 \%$ of patients referred to pulmonary rehabilitation managed to complete the programmes [10]. Similar findings of poor patient engagement have also been reported across the pulmonary rehabilitation service pathway in quantitative and qualitative studies worldwide [11]. Recent observational evidence suggests that pulmonary rehabilitation in the acute setting presents the greatest challenge of patient acceptance, with completion rates $<15 \%[12,13]$. Major barriers to uptake and/or completion of pulmonary rehabilitation in a stable or acute setting include travel and transport, perception of illness, lack of perceived benefit from attendance, disruption to routine, inconvenient timing, influence of general practitioner, illness and comorbidities, current smoking, depression, and lack of social support [11-17].

At a national and international level, it is widely accepted that healthcare providers urgently need to respond to the challenges of patient uptake and completion of pulmonary rehabilitation by improving the access to and the quality of services [18, 19]. While some barriers limiting patient acceptance of pulmonary rehabilitation programmes may be overcome by improvement in access and quality of service provision, there is an onus on the wider respiratory research community to identify the potential interventions that can further advance evidence-based policy in pulmonary rehabilitation [18]. There is no systematic review of interventions for improving uptake and/or completion of pulmonary rehabilitation. There is a need for a robust synthesis of current evidence that will have maximum potential to inform clinical practice and the design of future trials that assess the efficacy of interventions in improving uptake and/or completion of pulmonary rehabilitation. Such a review is important to identify effective approaches for supporting patient engagement with a treatment recognised to be one of the most cost-effective treatments for COPD. The primary aim of this study was to systematically review the effects of interventions for improving patient uptake and completion of pulmonary rehabilitation.

\section{Methods}

Methods of analysis and inclusion criteria were specified in advance and documented in a protocol that was registered on PROSPERO (International Prospective Register of Systematic Reviews; www.crd.york.ac. uk/PROSPERO/) with identifier CRD42016038524.

\section{Selection criteria}

Studies were considered for inclusion in this systematic review according to the following criteria.

\section{Participants}

Adults with a diagnosis of COPD confirmed by spirometry (in line with national or international criteria, e.g. British Thoracic Society (BTS), American Thoracic Society (ATS)/European Respiratory Society (ERS) or Global Initiative for Chronic Obstructive Lung Disease (GOLD)) and referred to pulmonary rehabilitation. Where the aim of a study was to increase completion rate, participants are those referred and already enrolled on to pulmonary rehabilitation at start of study. 


\section{Intervention}

Any intervention with the specific aim to improve patient uptake and/or completion of pulmonary rehabilitation. Studies which aimed to improve patient uptake and/or completion of pulmonary rehabilitation via interventions targeted at partners, carers, family members or healthcare providers/health professionals who refer patients to/deliver pulmonary rehabilitation would also be included.

\section{Comparison}

Any concurrent control group referred to and/or enrolled on to pulmonary rehabilitation, but not receiving an intervention aimed to improve patient uptake and/or completion (i.e. not beyond usual care).

\section{Outcomes}

Uptake of pulmonary rehabilitation (received baseline assessment and/or enrolled on to pulmonary rehabilitation). Completion of pulmonary rehabilitation (received discharge assessment; total number of sessions attended).

\section{Study design}

Randomised controlled trials that involved allocation of participants at an individual or cluster level, or via a quasi-randomised method.

\section{Search strategy}

To identify any existing relevant systematic reviews we searched the Cochrane Database of Systematic Reviews, PROSPERO, and the Database of Abstracts of Reviews of Effects of the National Institute of Health Research (NIHR) Service Centre for Reviews and Dissemination. To identify primary studies, we searched the following bibliographic databases: MEDLINE, Embase, CINAHL (Cumulative Index to Nursing and Allied Health Literature), CENTRAL (Cochrane Central Register of Controlled Trials) and PEDro (Physiotherapy Evidence Database). We searched the trial registers ClinicalTrials.gov, Current Controlled trials, and Health Technology Assessment in progress of the NIHR Service Centre for Reviews and Dissemination. We also searched EThOS (British Library) and Conference Proceedings Citation Index (Web of Science Core Collection). All information sources were searched between March 24, 2016 and April 28, 2016. No limits on publication date or language were set. Key search terms were structured around the population (e.g. "Lung Diseases, Obstructive", "COPD"), intervention target (e.g. "exercise therapy", "rehabilitat*"), outcomes (e.g. "adherence", "attendance") and study type (e.g. "randomised" or "randomized", "random allocation"). A full electronic search strategy for one database is provided in the supplementary material. Database searching was supplemented by contact with study authors, experts and research groups, forward and backward citation tracking from included studies or review articles, and continued monitoring with internet searches (e.g. Google Scholar) until June 14, 2016.

Search results were compiled using EndNote (Clarivate Analytics, Philadelphia, PA, USA). Duplicate citations were removed, and titles and abstracts screened independently by two reviewers against the inclusion criteria. Full-text articles were retrieved and assessed by two reviewers when studies were not excluded based on title or abstract. Discrepancies were resolved through discussion and inclusion of a third reviewer.

\section{Data extraction and quality appraisal}

We developed, tested and refined a structured data collection form based on the Cochrane data extraction template for interventions. One reviewer extracted data, which was cross-checked for accuracy by a second reviewer. The following information was extracted: methods of study (date/title of study, aim of study, study design, unit of allocation, duration of study, duration of intervention, primary outcome, other outcomes, funding source), participants (age, sex, disease severity, comorbidities, inclusion criteria, exclusion criteria, method of recruitment of participants, total number randomised, clusters, baseline imbalances), intervention/comparator (group names, number randomised to group-sample size, delivery, content, timing, frequency, duration, providers, co-interventions) and outcomes (name/definition, type, unit of analysis, person measuring/reporting, missing participants/data, reasons missing).

Two reviewers independently assessed the risk of bias for any studies included in the review using the Cochrane Risk of Bias Table with the following domains: random sequence generation, allocation concealment, blinding (outcome assessors) incomplete outcome data, selective outcome reporting and other bias [20]. Each domain was classified as adequate, unclear or inadequate, with the overall risk of bias for the study classified using the following criteria: 1) low risk of bias (all criteria deemed adequate), 2) moderate risk of bias (one criterion graded inadequate or two graded unclear) and 3) high risk of bias (more than one criterion deemed inadequate or more than two graded unclear). A third reviewer was available to resolve any disagreements between the two reviewers. Due to the nature of the interventions, 
blinding of participants and personnel to treatment allocation was not expected, and hence blinding only applied to outcome assessors.

\section{Data analysis}

The primary measure of effect for uptake of pulmonary rehabilitation was defined as total number of participants in each group who received baseline assessment and attended at least one session of pulmonary rehabilitation. Total number of participants who received a baseline assessment but did not enrol on to pulmonary rehabilitation was a secondary summary measure. The primary measure for completion of pulmonary rehabilitation was defined as total number of participants who received a discharge (follow-up) assessment. All of these were treated as dichotomous outcomes and were interpreted as risk ratios. We also included the number of sessions of pulmonary rehabilitation attended by participants as a secondary summary measure. This was treated as count data and interpreted as rate ratios (total number of sessions attended by participant/total number of sessions prescribed to participant).

If there were a sufficient number of studies that overlapped in terms of outcome measures, we planned to pool these data together (with participant as the unit of analysis) in a meta-analysis using a generic inverse variance random effects method and explore any sources (e.g. clinical and/or methodological diversity) of heterogeneity identified by the $I^{2}$-test statistic. Due to the insufficient amount of evidence, we provide a narrative synthesis of our findings.

Our pre-specified sensitivity analyses included assessing the effect of removing included studies classified as medium or high risk of bias within the Cochrane Risk of Bias Table. We planned to carry out subgroup analysis on the primary measures of effect using the following: setting of pulmonary rehabilitation (hospital, community or home), severity of COPD (GOLD stages), timing of pulmonary rehabilitation $(<4$ or $>4$ weeks post-exacerbation), source of pulmonary rehabilitation referral (referrer and contact method) and study design (individually randomised versus cluster). However, there were too few studies to conduct the above analyses.

\section{Results}

After removal of duplicates, searching identified 2468 distinct citations. 2429 citations were excluded during the initial screening phase (titles and abstracts) (figure 1). For the remaining 39 citations, full-text papers

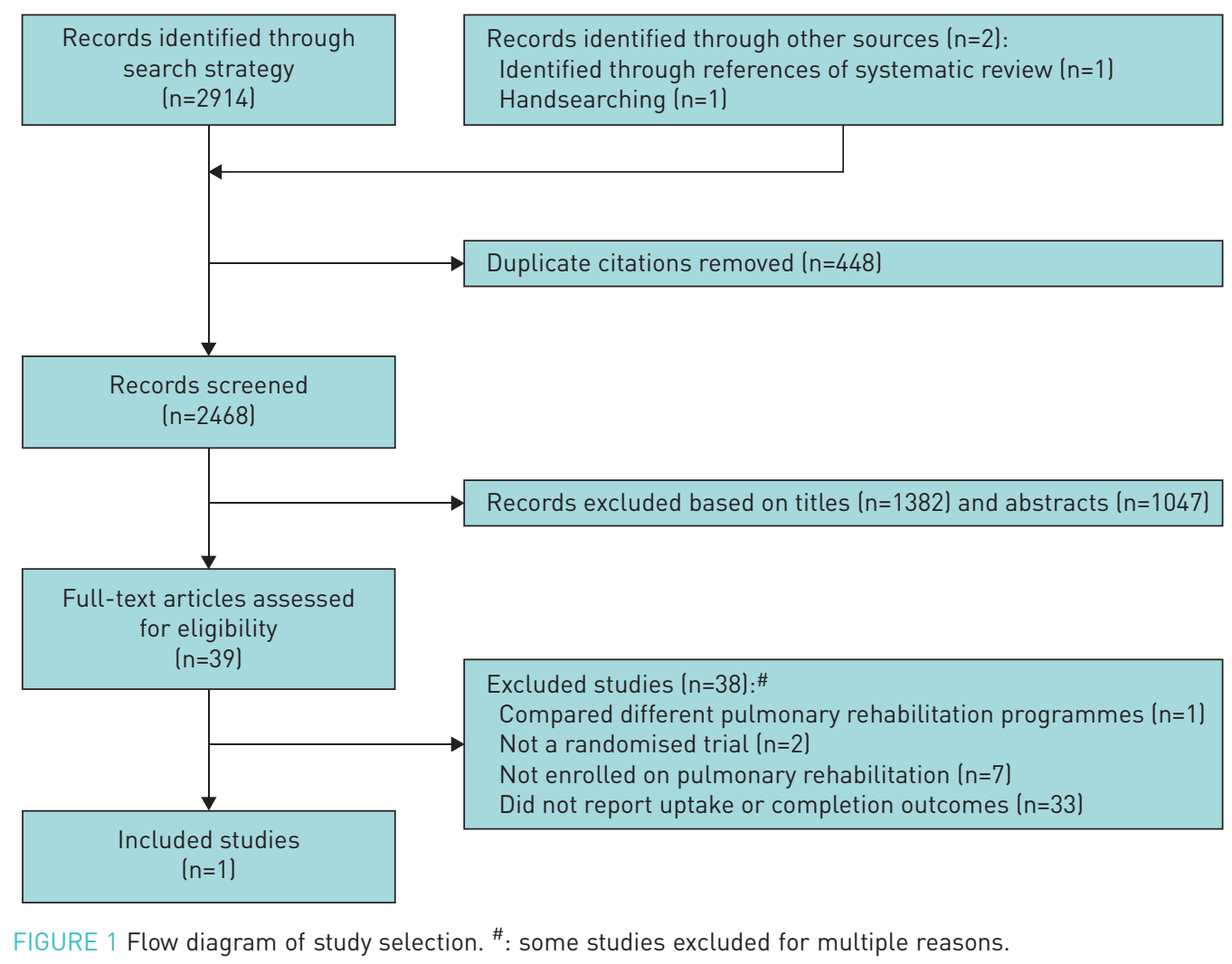




\begin{tabular}{|c|c|c|c|c|c|}
\hline Study (country) & Study design & Participants & Intervention & Comparison & Outcomes \\
\hline $\begin{array}{l}\text { RINGBAEK et al. [21] } \\
\text { (Denmark) }\end{array}$ & $\begin{array}{l}\text { Quasi-randomised } \\
\text { trial (allocation by } \\
\text { alternation) }\end{array}$ & $\begin{array}{l}\text { Intervention: } 46 \text { participants, } \\
\text { age } 68 \pm 9 \text { years, } 55 \% \\
\text { male, BMI } 24 \pm 5 \mathrm{~kg} \cdot \mathrm{m}^{-2}, \\
\text { FEV } 131 \pm 9 \% \text { predicted, } \\
\text { current smokers } 14 \% \text {, } \\
\text { long-term oxygen therapy } 7 \% \text {, } \\
\text { CAT score } 20 \pm 7 \text {, } \\
\text { MRC dyspnoea } \\
\text { score } 4 \text { (2-5). } \\
\text { Control: } 69 \text { participants, age } \\
69 \pm 11 \text { years, } 39 \% \text { male, BMI } \\
25 \pm 6 \mathrm{~kg} \cdot \mathrm{m}^{-2}, \text { FEV } 135 \pm 11 \% \\
\text { predicted, current smokers } \\
17 \%, \text { long-term oxygen } \\
\text { therapy } 13 \%, \text { CAT score } 19 \pm 6 \text {, } \\
\text { MRC dyspnoea } \\
\text { score } 4(2-5) .\end{array}$ & $\begin{array}{l}\text { Use of a wireless tablet computer } \\
\text { (Nexus 7) with mobile-based } \\
\text { internet connectivity plus usual } \\
\text { care. The tablet software } \\
\text { consisted of 1) a training diary } \\
\text { (including the type, frequency, } \\
\text { duration of exercises, and } \\
\text { registered dyspnoea after each } \\
\text { exercise using the Borg } \\
\text { dyspnoea score), } \\
\text { 2) video recordings of all the } \\
\text { training exercises used and 3) } \\
\text { training results for patients to } \\
\text { monitor their own training. Staff } \\
\text { had access to this information, } \\
\text { and used it discuss progress, } \\
\text { individual barriers and } \\
\text { opportunities as well as to } \\
\text { encourage further training. }\end{array}$ & $\begin{array}{l}\text { Usual care: pulmonary } \\
\text { rehabilitation twice weekly for } \\
7 \text { weeks at one of two hospitals } \\
\text { (Gentofte Hospital and Hvidovre } \\
\text { Hospital) or } 10 \text { weeks at } \\
\text { Nordsjælland Hospital with } \\
\text { each session lasting } 2 \mathrm{~h} \text {. Each } \\
\text { session consisted of } 1 \mathrm{~h} \text { of } \\
\text { supervised exercise and } 1 \mathrm{~h} \text { of } \\
\text { education. The supervised } \\
\text { training sessions consisted of } \\
\text { walking and cycling. Patients } \\
\text { were instructed to exercise at a } \\
\text { level equal to } 85 \% \text { of predicted } \\
\text { peak oxygen uptake as } \\
\text { calculated from the } \\
\text { incremental shuttle walk test. }\end{array}$ & $\begin{array}{l}\text { Dropout rates. } \\
\text { 1) Completion of rehabilitation: } \\
\text { intervention } 42 \text { out of } 46 \\
\text { participants; control } 57 \text { out of } 69 \\
\text { participants. 2) Sessions } \\
\text { attended: intervention } 631 \text { out of } \\
784 \text { sessions; control } 509 \text { out of } \\
724 \text { sessions. }\end{array}$ \\
\hline
\end{tabular}


were obtained and subsequently assessed independently against the eligibility criteria by two reviewers with discrepancies resolved by discussion with a third reviewer. One study (2.5\%) met the inclusion criteria [21] and had extractable data on an intervention promoting completion of pulmonary rehabilitation (table 1). The most common reason for exclusion of full-text papers was no measure of uptake or completion of pulmonary rehabilitation. A full list of excluded studies, together with reasons for exclusion, can be found in the supplementary material.

The one study included was published in 2016 [21]. The trial was conducted within secondary care (pulmonary clinics) in Denmark and included 115 participants (42\% males). The inclusion criteria for entry into were a diagnosis of stable COPD (confirmed spirometry as forced expiratory volume in $1 \mathrm{~s}$ $\left(\mathrm{FEV}_{1}\right)<80 \%$ predicted and $\mathrm{FEV}_{1} /$ forced vital capacity $<70 \%$ predicted) and enrolment on to a pulmonary rehabilitation programme at one of three hospitals. Exclusion criteria for the study were any musculoskeletal, cardiac and cognitive disorders that limited the ability of the participants to train and attend classes. A total of 146 participants were originally referred to pulmonary rehabilitation during the study period, but 14 were excluded (no COPD diagnosis) and 17 refused to participate in the study.

This trial compared pulmonary rehabilitation plus use of a tablet computer with instructions and a training diary (intervention group) to a pulmonary rehabilitation only group. Every second training group was allocated to use a 7-inch tablet computer (Nexus 7; Asus, Taipei, Taiwan) and thus met our criteria of study type of a quasi-randomised trial (i.e. alternation). The trial design did not involve any blinding. The intervention lasted for the duration of pulmonary rehabilitation. Both groups included provision of pulmonary rehabilitation for 7-10 weeks. The investigators hypothesised that, by providing a common platform for cooperation between the patient and the health professionals, the intervention could empower the patient to become an active participant evaluating his/her own improvements.

The intervention and the control group did not achieve comparable changes in the primary outcome measures (endurance shuttle walk test (ESWT) and COPD Assessment Test (CAT)) following pulmonary rehabilitation. The mean change in walking time (ESWT) was significantly better in the control group compared with the intervention group, with this difference also being greater than the proposed minimal clinical important difference (45-85 s) [22]. Although it was reported that the change in CAT score after pulmonary rehabilitation did not differ significantly between groups, the intervention group demonstrated a reduction that is considered to be clinically important ( 2 units) [23], while there was a modest increase in CAT score in the control group.

Dropout rates (completion of pulmonary rehabilitation) were not specified as a primary outcome, but rather labelled as a secondary effect parameter. Completion was defined as attending the evaluation visit (assessment at end of pulmonary rehabilitation). Completion of pulmonary rehabilitation was treated as discrete data and interpreted as a difference in group proportions. On request, authors of the study were able to provide data on the number of sessions of pulmonary rehabilitation attended within study groups.

The point estimate of effect for the included trial indicated greater completion of pulmonary rehabilitation among participants allocated to the intervention group compared with those allocated to the usual care control group (risk ratio 1.11, 95\% CI 0.96-1.28) (table 1). The difference in completion rates between groups did not achieve statistical significance in the study. The rate of attendance at prescribed sessions was greater in the intervention compared with the control group (rate ratio 1.15, 95\% CI 1.14-1.16) (table 1). The included study was, however, considered to have a high overall risk of bias (table 2). Four (67\%) of the individual risk of bias domains were graded inadequate. This was mostly due to the nonblinded, quasi-randomised allocation nature of the study.

We identified three study protocols describing at least three randomised trials (based in the UK) that meet our eligibility criteria [24-26]. One of these is assessing the feasibility of a definitive randomised controlled trial that will assess whether in-hospital exercise and in-home rehabilitation delivered in isolation or concurrently following an acute exacerbation of COPD are clinically and cost-effective, including their impact on subsequent attendance at community group-based pulmonary rehabilitation [24]. Another trial is evaluating uptake to initial assessment of post-exacerbation pulmonary rehabilitation (primary outcome measure) in response to a brief patient-designed educational video provided before invitation to the programme [25]. The other protocol details a seamless three-phase study (phase I: preparation; phase II: randomised pilot; phase III: a fully powered multicentre, individually randomised controlled trial) [26]. The aim is to determine the effect of a psychological intervention combined with practical problem solving (that links and interdigitates with usual pulmonary rehabilitation) on the Hospital Anxiety and Depression Scale in COPD. Contact with an investigator of this study and the other two protocols suggested data would be available from each of these studies to determine the efficacy of the interventions on all of the pre-specified outcome measures of this review. Additional details of the studies are provided in table 3. 


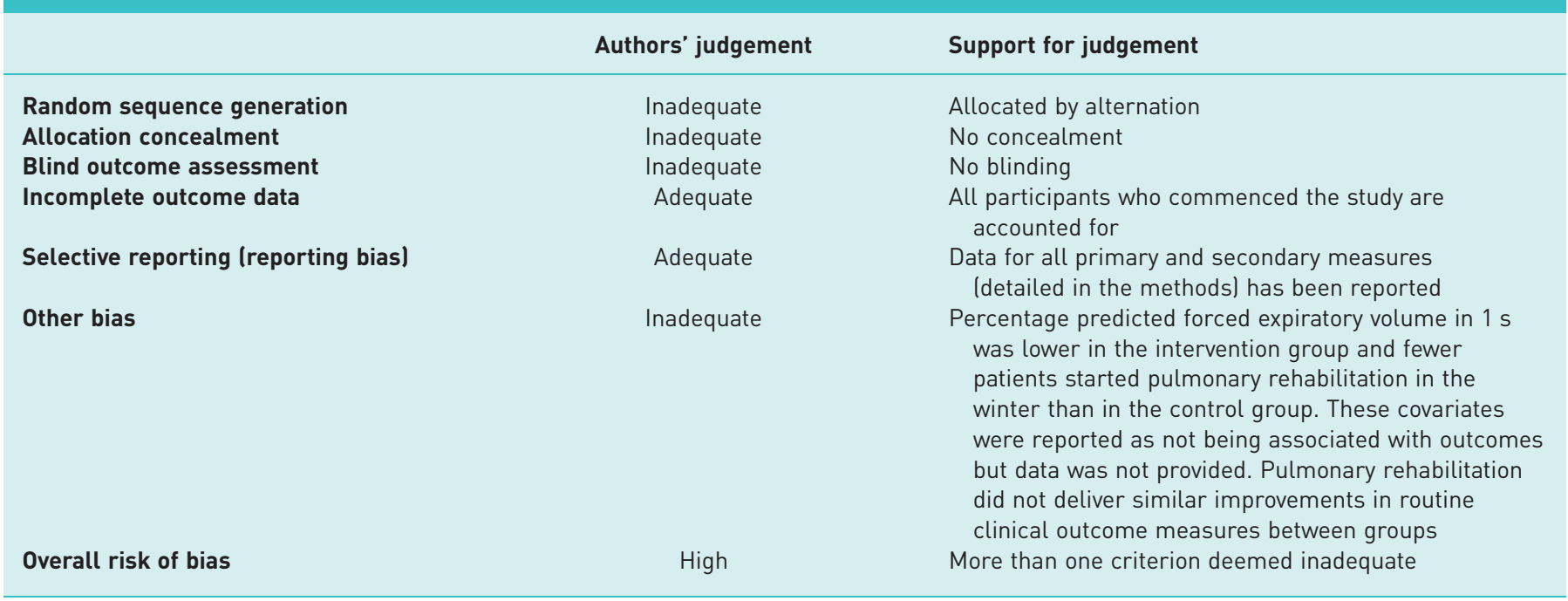

\section{Discussion}

The review identified only one quasi-randomised controlled trial assessing the efficacy of an intervention to improve completion of pulmonary rehabilitation [21]. The intervention was the allocation of a tablet computer (equipped with training diary, exercise videos and training results) to patients undergoing outpatient pulmonary rehabilitation. Synthesis of the available data from this trial suggested that the intervention had a small (but not statistically significant) effect in which, compared with usual care, use of the tablet computer increased the likelihood for completion of pulmonary rehabilitation by $11 \%$. This trial must be interpreted in relation to the quality of the evidence. As a secondary effect parameter, the study was underpowered to detect a significant difference between groups in completion rates. Further data, provided by the study authors, also indicated a greater rate of session attendance throughout pulmonary rehabilitation with use of the tablet computer. It is important to note that the major limitations in design of the study (lack of concealed allocation, blinding) may confer a biased assessment of the intervention effect.

\section{Strengths and limitations of the study}

This review adhered to the pre-specified protocol using appropriate methods to select, evaluate and synthesise all the relevant evidence. A comprehensive search for published and unpublished studies, which included multiple electronic databases, scanning of bibliographies and contact with authors, yielded only one published study. A strength of this review is that it presents the first attempt to collate and synthesise the effects of interventions for improving patient uptake and completion of pulmonary rehabilitation. We had strict definitions on outcome measures for the purpose of this review and did not consider other outcomes, such as amount or frequency of unsupervised exercise/physical activity during or after pulmonary rehabilitation. Some of the excluded studies did include interventions targeted at these outcomes, but our primary interest was in those studies aiming to address the commonly reported poor patient uptake and completion of pulmonary rehabilitation in COPD.

The lack of identification, and hence inclusion, of unpublished evidence is often considered a limitation in systematic reviews. Effects estimated from published studies may be inflated due to bias towards the nonpublication of studies with nonsignificant effects. However, the identification of only one published study [21] with nonsignificant findings mitigates concerns about publication bias. Rather, this review reveals major limitations in the available randomised controlled evidence in the area.

\section{Comparison with other studies}

There is currently no systematic review that has selected and critically appraised all of the randomised trial evidence on interventions to improve uptake and completion of pulmonary rehabilitation. We are aware of reviews evaluating the effect of interventions to improve patient uptake and adherence of rehabilitation in cardiac populations [27]. Previous joint ATS/ERS statements and the BTS pulmonary rehabilitation guidelines $[5,28]$ identified one study that improved patient completion of pulmonary rehabilitation via a group-based session incorporating cognitive behavioural techniques [29]. This, however, was a nonrandomised study with historical controls and the evidence was graded to have a high risk of 
TABLE 3 Characteristics of ongoing studies

\begin{tabular}{|c|c|c|c|c|c|c|c|}
\hline Study name or title & Chief investigator & $\begin{array}{l}\text { Study period } \\
\text { (start and end } \\
\text { dates) } \\
\text { (country) }\end{array}$ & Study design & Participants & Intervention & Comparison & Outcomes \\
\hline $\begin{array}{l}\text { Pulmonary Rehabilitation } \\
\text { and ACTlvity after COPD } \\
\text { Exacerbations: the } \\
\text { PRACTICE trial: a } \\
\text { randomised trial [24] }\end{array}$ & R. Hughes & $\begin{array}{l}\text { January } 2015 \text { to } \\
\text { December } 2016 \\
\text { (UK) }\end{array}$ & $\begin{array}{l}\text { Parallel group, } \\
\text { randomised pilot } \\
2 \times 2 \text { trial }\end{array}$ & $\begin{array}{l}76 \text { participants with } \\
\text { clinically identified } \\
\text { exacerbation of } \\
\text { diagnosed COPD }\end{array}$ & $\begin{array}{l}\text { 1) In-hospital exercise } \\
\text { training followed by } \\
\text { in-home post-discharge } \\
\text { early rehabilitation; } \\
\text { 2) standard in-hospital } \\
\text { care followed by in-home } \\
\text { post-discharge early } \\
\text { rehabilitation }\end{array}$ & $\begin{array}{l}\text { 1) In-hospital exercise } \\
\text { training followed by } \\
\text { standard discharge care; } \\
\text { 2) standard in-hospital } \\
\text { care followed by } \\
\text { standard discharge }\end{array}$ & $\begin{array}{l}\text { Subsequent uptake, } \\
\text { adherence and } \\
\text { completion of } \\
\text { group-based community } \\
\text { pulmonary rehabilitation }\end{array}$ \\
\hline $\begin{array}{l}\text { Video to Increase } \\
\text { RehabiliTation Uptake } \\
\text { following hospitalised } \\
\text { Exacerbations of COPD } \\
\text { (VIRTUE): a randomised } \\
\text { controlled trial [25] }\end{array}$ & W. Man & $\begin{array}{l}\text { January } 2015 \text { to } \\
\text { January } 2017 \\
\text { (UK) }\end{array}$ & $\begin{array}{l}\text { Parallel group, } \\
\text { randomised trial }\end{array}$ & $\begin{array}{l}200 \text { adults aged }>40 \text { years } \\
\text { diagnosed with COPD, } \\
\text { admitted to hospital with } \\
\text { acute exacerbation of } \\
\text { COPD and fit enough to } \\
\text { take part in pulmonary } \\
\text { rehabilitation }\end{array}$ & $\begin{array}{l}\text { Participants will be asked } \\
\text { to watch a 5-min } \\
\text { patient-designed video } \\
\text { promoting early } \\
\text { pulmonary rehabilitation } \\
\text { plus usual care }\end{array}$ & $\begin{array}{l}\text { Usual care: provided with } \\
\text { standard verbal } \\
\text { information and an A5 } \\
\text { patient information } \\
\text { leaflet about early } \\
\text { pulmonary rehabilitation }\end{array}$ & $\begin{array}{l}\text { Uptake, adherence and } \\
\text { completion of } \\
\text { post-exacerbation } \\
\text { pulmonary rehabilitation }\end{array}$ \\
\hline $\begin{array}{l}\text { A tailored, psychological } \\
\text { intervention for mild to } \\
\text { moderate anxiety or } \\
\text { depression in people } \\
\text { with COPD: Tailored } \\
\text { intervention for ANxiety } \\
\text { and DEpression } \\
\text { Management in COPD } \\
\text { (TANDEM] [26] }\end{array}$ & S. Taylor & $\begin{array}{l}\text { April } 2016 \text { to } \\
\text { January } 2021 \\
\text { (UK) }\end{array}$ & $\begin{array}{l}\text { Three-phase study } \\
\text { (phase I: } \\
\text { preparation; } \\
\text { phase II: pilot } \\
\text { randomised } \\
\text { controlled trial; } \\
\text { phase III: a fully } \\
\text { powered } \\
\text { individually } \\
\text { randomised } \\
\text { controlled trial) }\end{array}$ & $\begin{array}{l}\text { Adults with } \\
\text { moderate-severe COPD } \\
\text { with mild-moderate } \\
\text { comorbid anxiety or } \\
\text { depression and eligible } \\
\text { for attendance at their } \\
\text { local pulmonary } \\
\text { rehabilitation; phase II: } \\
45 \text { participants; phase } \\
\text { III: } 430 \text { participants }\end{array}$ & $\begin{array}{l}\text { Tailored, one-to-one } \\
\text { psychological } \\
\text { intervention combined } \\
\text { with practical } \\
\text { problem-solving } \\
\text { components based on: } \\
\text { Self-management } \\
\text { Programme of Activity } \\
\text { Coping and Education } \\
\text { (SPACE) and The Lung } \\
\text { Manual, a nurse-led } \\
\text { intervention based on } \\
\text { cognitive behavioural } \\
\text { principles and } \\
\text { self-management, and } \\
\text { developed to address } \\
\text { mood disorders of } \\
\text { anxiety in COPD; this } \\
\text { psychological } \\
\text { intervention links into, } \\
\text { and interdigitates with, } \\
\text { usual pulmonary } \\
\text { rehabilitation, but is } \\
\text { independent of it }\end{array}$ & $\begin{array}{l}\text { Usual care: standard } \\
\text { pulmonary rehabilitation } \\
\text { without interdigitating } \\
\text { psychological } \\
\text { intervention }\end{array}$ & $\begin{array}{l}\text { Uptake and completion of } \\
\text { pulmonary rehabilitation } \\
\text { (to be informed by the } \\
\text { pre-pilot and pilot } \\
\text { studies) }\end{array}$ \\
\hline
\end{tabular}


confounding, bias or chance and a significant risk that the relationship was not causal. Other reviews have included quantitative studies and qualitative studies of attendance and adherence to pulmonary rehabilitation but mainly focused on the identification of the barriers, not the evaluation of strategies to address them $[11,16]$. Systematic reviews of randomised trials assessing add-on/supplemental interventions to pulmonary rehabilitation are also available, but the measures are limited to routine clinical outcomes only (health-related quality of life and exercise capacity) [30, 31].

\section{Implications for clinical practice and research}

No recommendations for this crucial area of practice can be made given the current lack of evidence. Application of an intervention with demonstrated efficacy will have important implications for clinical practice. It is somewhat surprising to see a lack of simple practical interventions to support decision making within the pulmonary rehabilitation service pathway. Poor patient uptake and completion in conjunction with existing low referral rates of pulmonary rehabilitation imply a major failure in the healthcare system [18]. The potential for these issues to undermine the effectiveness of pulmonary rehabilitation, and put further burden on increasingly stringent financial resources, places emphasis on this area as a research priority.

There is a strong rationale for enhancing patient uptake and completion of pulmonary rehabilitation but further high-quality research is needed, particularly single-intervention strategies based on theoretical frameworks or tailored to the key barriers that are relevant to the setting. We have identified at least three ongoing randomised trials in the UK that will provide important new evidence, but only one of these studies defines uptake or completion as a primary measure [25], and hence it is unlikely these studies alone will lead to changes in practice nationally or internationally. We reiterate, with urgency, calls from the 2013 BTS pulmonary rehabilitation guideline for robust, well-designed trials evaluating techniques before and during pulmonary rehabilitation [28]. While we understand there will be interest in assessing the long-term effects of interventions that improve patient compliance (e.g. clinical outcomes), current focus should be placed on delivering short-term studies that evaluate more immediate impact on patients (e.g. uptake of pulmonary rehabilitation).

Despite wide awareness of patient barriers to pulmonary rehabilitation, affirmed most recently by STEINER and RoBERTs [10], our review highlights a concerning under-appreciation of intervention trials in this area. Numerous qualitative studies and editorials have previously proposed potential interventions, but none of these have been evaluated in a randomised trial context [32-39]. Conceivably, excluded studies in this review consisted of interventions that when tested within an appropriate designed, adequately powered trial may have a positive effect on uptake or completion of pulmonary rehabilitation. We also recognise the calls for further studies on novel approaches to pulmonary rehabilitation (e.g. home-based, web-based, telehealth support programmes) that were not considered for inclusion in this review, but may have the potential to influence patient compliance through provision of a more acceptable setting.

It is reasonable to suggest that rehabilitation service providers may have previously attempted to overcome challenges of patient uptake and completion by implementing changes without formal evaluation. Recommendations have recently been made at both the national and international level for improvement in access and quality of service provision of pulmonary rehabilitation [10, 19]. We feel this approach alone will not maximise the opportunity to address the challenges of patient uptake and completion. The completion of randomised trials will not only provide a robust platform to evaluate a range of interventions (similar to which pulmonary rehabilitation itself is founded upon), but also provide clearer pathways for the sharing of evidence within the wider respiratory communities. Interventions should be developed with the timing and setting of pulmonary rehabilitation in mind, and whether they are best targeted at all COPD patients or specific clinical phenotypes. This is pertinent for other chronic respiratory conditions that are also referred to pulmonary rehabilitation.

\section{Conclusions}

With only one low-quality quasi-randomised trial included, there is insufficient evidence to guide clinical practice with regard to interventions for improving patient uptake and completion of pulmonary rehabilitation in COPD. The paucity of evidence in this area acts as a major limitation for any healthcare services planning to address continuing challenges around patient uptake/completion of pulmonary rehabilitation. Despite increasing awareness of patient barriers to pulmonary rehabilitation, our review highlights the existing under-appreciation of intervention trials in this area and is an urgent call for more evidence to guide the pulmonary rehabilitation service pathway. This knowledge gap should be viewed as an area of research priority due to its likely impact in undermining wider implementation of pulmonary rehabilitation and restricting patient access to a treatment considered the cornerstone of COPD. Future 
research should utilise high-quality study designs that aim to address the major modifiable barriers to uptake and completion.

\section{Acknowledgements}

We would like to acknowledge the support of colleagues at Lincolnshire Community Health Services NHS trust (Liz Hill, David Bareham and Janice Wiseman), Leicestershire Partnership NHS Trust (Alex Woodward), Care Plus Group (Kylie Farbrace), Northern Lincolnshire and Goole NHS Foundation Trust (Joanne Richardson), United Lincolnshire Hospitals NHS Trust (Dave Clifton), National Institute of Health Research Clinical Research Network (Debbie Jeffrey), University of Lincoln (Alex Jenkins, Ffion Curtis and Neil Holden), and University of Nottingham (Neil Coulson) in conception of the review question as part of the development of the Lincolnshire Joint Research Agenda on COPD. The authors would like to thank Thomas Ringbaek (Respiratory Dept, Hvidovre Hospital, Copenhagen, Denmark) for providing data upon request for this systematic review and investigators (Rodney Hughes [24], William Man [25] and Karen Heslop-Marshall [26]) within their respective ongoing trials for their correspondence. We also thank the reviewers of this manuscript for their constructive comments, the addressing of which has improved the manuscript.

\section{References}

1 Global Initiative for Chronic Obstructive Lung Disease. Global Strategy for Diagnosis, Management, and Prevention of COPD. 2016. www.goldcopd.org Date last accessed: January 28, 2016.

2 Lozano R, Naghavi M, Foreman K, et al. Global and regional mortality from 235 causes of death for 20 age groups in 1990 and 2010: a systematic analysis for the Global Burden of Disease Study 2010. Lancet 2012; 380: 2095-2128.

3 Murray CJ, Lopez AD. Measuring the global burden of disease. N Engl J Med 2013; 369: 448-457.

4 Department of Health. An outcomes strategy for chronic obstructive pulmonary disease (COPD) and asthma in England: NHS companion document. www.gov.uk/government/publications/an-outcomes-strategy-for-peoplewith-chronic-obstructive-pulmonary-disease-copd-and-asthma-in-england Date last accessed: January 28, 2016.

5 Spruit MA, Singh SJ, Garvey C, et al. An official American Thoracic Society/European Respiratory Society statement: key concepts and advances in pulmonary rehabilitation. Am J Respir Crit Care Med 2013; 188: 13-64.

6 McCarthy B, Casey D, Devane D, et al. Pulmonary rehabilitation for chronic obstructive pulmonary disease. Cochrane Database Syst Rev 2015; 2: CD003793.

7 Lacasse Y, Cates CJ, McCarthy B, et al. This Cochrane Review is closed: deciding what constitutes enough research and where next for pulmonary rehabilitation in COPD. Cochrane Database Syst Rev 2015; 18: ED000107.

8 Puhan MA, Gimeno-Santos E, Scharplatz M, et al. Pulmonary rehabilitation following exacerbations of chronic obstructive pulmonary disease. Cochrane Database Syst Rev 2011; 10: CD005305.

9 Wise J. NICE recommends pulmonary rehabilitation programmes for patients with COPD. BMJ 2016; 352: i768.

10 National COPD Audit Programme: Pulmonary Rehabilitation Workstream. Pulmonary Rehabilitation: Steps to Breathe Better. London, Royal College of Physicians, 2016.

11 Keating A, Lee A, Holland AE. What prevents people with chronic obstructive pulmonary disease from attending pulmonary rehabilitation? A systematic review. Chron Respir Dis 2011; 8: 89-99.

12 Jones SE, Green SA, Clark AL, et al. Pulmonary rehabilitation following hospitalisation for acute exacerbation of COPD: referrals, uptake and adherence. Thorax 2014; 69: 181-182.

13 Harrison SL, Robertson N, Graham CD, et al. Can we identify patients with different illness schema following an acute exacerbation of COPD: a cluster analysis. Respir Med 2014; 108: 319-328.

14 Cassidy S, Turnbull S, Gardani M, et al. Attendance at pulmonary rehabilitation classes: an exploration of demographic, physiological and psychological factors that predict completion of treatment. Chron Respir Dis 2014; 11: 95-102.

15 Hayton C, Clark A, Olive S, et al. Barriers to pulmonary rehabilitation: characteristics that predict patient attendance and adherence. Respir Med 2013; 107: 401-407.

16 Sohanpal R, Steed L, Mars T, et al. Understanding patient participation behaviour in studies of COPD support programmes such as pulmonary rehabilitation and self-management: a qualitative synthesis with application of theory. NPJ Prim Care Respir Med 2015; 25: 15054.

17 Brown AT, Hitchcock J, Schumann C, et al. Determinants of successful completion of pulmonary rehabilitation in COPD. Int J Chron Obstruct Pulmon Dis 2016; 11: 391-397.

18 Steiner MC, Roberts CM. Pulmonary rehabilitation: the next steps. Lancet Respir Med 2016; 4: 172-173.

19 Rochester CL, Vogiatzis I, Holland AE, et al. An Official American Thoracic Society/European Respiratory Society policy statement: enhancing implementation, use, and delivery of pulmonary rehabilitation. Am J Respir Crit Care Med 2015; 192: 1373-1386.

20 Higgins JPT, Green S, eds. Cochrane Handbook for Systematic Reviews of Interventions Version 5.0.2 [updated September 2009]. The Cochrane Collaboration. http://handbook.cochrane.org/v5.0.2/ Date last accessed: February 22, 2016.

21 Ringbaek TJ, Lavesen M, Lange P. Tablet computers to support outpatient pulmonary rehabilitation in patients with COPD. Eur Clin Respir J 2016; 3: 31016.

22 Jones PW, Beeh KM, Chapman KR, et al. Minimal clinically important differences in pharmacological trials. Am J Respir Crit Care Med 2014; 189: 250-255.

23 Kon SS, Canavan JL, Jones SE, et al. Minimum clinically important difference for the COPD Assessment Test: a prospective analysis. Lancet Respir Med 2014; 2: 195-203.

24 Pulmonary Rehabilitation and ACTIvity after COPD Exacerbations: the PRACTICE Trial: a randomised trial. www.nets.nihr.ac.uk/projects/hta/132403 Date last accessed: April 28, 2016.

25 Video to Increase RehabiliTation Uptake following hospitalised Exacerbations of COPD (VIRTUE): a randomised controlled trial. www.isrctn.com/ISRCTN13165073 Date last accessed: April 28, 2016.

26 A tailored, psychological intervention for mild to moderate anxiety or depression in people with COPD: Tailored intervention for ANxiety and DEpression Management in COPD (TANDEM). www.nets.nihr.ac.uk/projects/hta/ 1314602 Date last accessed: June 6, 2016. 
27 Karmali KN, Davies P, Taylor F, et al. Promoting patient uptake and adherence in cardiac rehabilitation. Cochrane Database Syst Rev 2014; 6: CD007131.

28 Bolton CE, Bevan-Smith EF, Blakey JD, et al. British Thoracic Society guideline on pulmonary rehabilitation in adults. Thorax 2013; 68: 1-30.

29 Graves J, Sandrey V, Graves T, et al. Effectiveness of a group opt-in session on uptake and graduation rates for pulmonary rehabilitation. Chron Respir Dis 2010; 3: 159-164.

30 Camillo CA, Osadnik CR, van Remoortel H, et al. Effect of "add-on" interventions on exercise training in individuals with COPD: a systematic review. ERJ Open Res 2016; 2: 00078-2015.

31 Puhan MA, Schünemann HJ, Frey M, et al. Value of supplemental interventions to enhance the effectiveness of physical exercise during respiratory rehabilitation in COPD patients. A systematic review. Respir Res 2004; 5: 25.

32 Arnold E, Bruton A, Ellis-Hill C. Adherence to pulmonary rehabilitation: a qualitative study. Respir Med 2006; 100: 1716-1723.

33 Bulley C, Donaghy M, Howden S, et al. A prospective qualitative exploration of views about attending pulmonary rehabilitation. Physiother Res Int 2009; 14: 181-192.

34 Foster F, Piggott R, Riley L, et al. Working with primary care clinicians and patients to introduce strategies for increasing referrals for pulmonary rehabilitation. Prim Health Care Res Dev 2016; 17: 226-237.

35 Guo SE, Bruce A. Improving understanding of and adherence to pulmonary rehabilitation in patients with COPD: a qualitative inquiry of patient and health professional perspectives. PLoS One 2014; 9: e110835.

36 Harris D, Hayter M, Allender S. Improving the uptake of pulmonary rehabilitation in patients with COPD: qualitative study of experiences and attitudes. Br J Gen Pract 2008; 58: 703-710.

37 Johnston KN, Young M, Grimmer KA, et al. Barriers to, and facilitators for, referral to pulmonary rehabilitation in COPD patients from the perspective of Australian general practitioners: a qualitative study. Prim Care Respir J 2013; 22: 319-324.

38 Spruit MA, Franssen FM. What to do before pulmonary rehabilitation to improve adherence? Chron Respir Dis 2010; 7: 131-133.

39 Williams JE. The challenge of increasing uptake of pulmonary rehabilitation: what can we do to maximise the chances of success? Chron Respir Dis 2011; 8: 87-88. 